\title{
Noise in homodyne detection
}

\author{
Bonny L. Schumaker \\ California Institute of Technology, Pasadena, California 91125
}

Received October 11, 1983; accepted February 22, 1984

\begin{abstract}
A simple but rigorous analysis of the important sources of noise in homodyne detection is presented. Output noise and signal-to-noise ratios are compared for direct detection, conventional (one-port) homodyning, and two-port homodyning, in which one monitors both output ports of a 50-50 beam splitter. It is shown that two-port homodyning is insensitive to local-oscillator quadrature-phase noise and hence provides (1) a means of detecting reduced quadrature-phase fluctuations (squeezing) that is perhaps more practical than one-port homodyning and (2) an output signal-to-noise ratio that can be a modest to significant improvement over that of one-port homodyning and direct detection.
\end{abstract}

It has been known for some time that phase-sensitive detection schemes, such as homodyning and heterodyning, provide a means of measuring one of a signal's two quadratures. ${ }^{1-3}$ Recently a clever scheme, referred to in this Letter as two-port homodyning, was proposed by Yuen and Chan (see Fig. 1) ${ }^{4,5}$; this scheme permits direct observation of one of the input signal's quadratures without placing such rigorous demands on local-oscillator (LO) performance as does conventional one-port homodyning. Hence the two-port scheme might provide a more practical means of observing the phenomenon of squeezing, ${ }^{6-8}$ in which the noise in one quadrature phase of a signal is reduced below the level required by quantum mechanics of a coherent state. This noise can be observed because the output noise in two-port homodyning can be made insensitive to all LO quadrature-phase noise, provided the $\mathrm{LO}$ power is much larger than the input-signal power. In this Letter I compare output noise and signal-to noise ratios for three different detection schemes-one-port homodyning, two-port homodyning, and direct detection.

A single plane-wave mode of the electromagnetic field at frequency $\Omega_{s}$ (the input-signal frequency) has an electric-field operator described by

$$
\begin{aligned}
E(t, x) & \propto 1 / 2\left\{a \exp \left[-i \Omega_{s}(t-x)\right]+a^{\dagger} \exp \left[i \Omega_{s}(t-x)\right]\right\} \\
& =a_{1} \cos \Omega_{s}(t-x)+a_{2} \sin \Omega_{s}(t-x)
\end{aligned}
$$

Here $a \equiv a_{1}+i a_{2}$ is the annihilation operator for the mode and $a_{1}$ and $a_{2}$ are its Hermitian quadrature-phase amplitudes. Homodyne schemes use a beam splitter to combine an input-signal field with a LO field of large power compared with the input-signal field (Fig. 1). The dominant signal-carrying term in the intensities from each of the two beam-splitter output ports is proportional to the mean field of that quadrature of the input-signal field $\left(a_{1}\right.$, say) that is in phase with the LO mean field after the beam splitter. One-port homodyne schemes use a photodetector to monitor the intensity from one output port of the beam splitter. Two-port homodyne schemes monitor the intensities from both output ports of a 50-50 beam splitter and then subtract the two photodetector outputs.
Each quadrature of the input-signal field and the LO field has associated with it a certain amount of noise (fluctuations), characterized by the variance of its quadrature-phase amplitude, i.e., $\Delta a_{1}^{2} \equiv\left\langle\left(a_{1}-\left\langle a_{1}\right\rangle\right)^{2}\right\rangle$ and $\Delta a_{2}{ }^{2}$ for the input-signal field. Quantum mechanics requires that $\Delta a_{1}{ }^{2} \Delta a_{2}{ }^{2} \geq 1 / 16$. Coherent states and the vacuum state have $\Delta a_{1}{ }^{2}=\Delta a_{2}{ }^{2}=1 / 4$ (random-phase noise). Single-mode squeezed states ${ }^{6-8}$ satisfy $\Delta a^{\prime}{ }_{1}{ }^{2} \Delta a^{\prime}{ }_{2}{ }^{2}=1 / 16$ but have $\Delta a^{\prime}{ }_{1}{ }^{2}<1 / 4$, where $a_{1}^{\prime}+i a_{2}^{\prime} \equiv e^{-i \varphi}\left(a_{1}+i a_{2}\right), 0 \leq \varphi<\pi$.

Aside from that added by nonideal photodetectors, the dominant output noise in homodyning comes from interference between the (large) LO mean field and the noise in the in-phase quadratures of the input-signal and LO fields ( $a_{1}$ and $b_{1}$, say). In direct detection the output noise reflects only intensity fluctuations in the input signal $\left[\Delta N_{a}{ }^{2} \equiv\left\langle\left(N_{a}-\left\langle N_{a}\right\rangle\right)^{2}\right\rangle\right]$, but in homodyning it reflects the variances $\Delta a_{1}{ }^{2}$ and $\Delta b_{1}{ }^{2}$. In one-port homodyning with a lossless beam splitter of power transmissivity $T$ the dominant output noise is proportional to the sum $T \Delta a_{1}{ }^{2}+(1-T) \Delta b_{1}^{2}$; in twoport homodyning it can be made proportional to $\Delta a_{1}{ }^{2}$ alone. The two-port scheme can yield a better output signal-to-noise ratio (SNR) than both direct detection and one-port homodyning, the improvement over the latter being most significant when $\Delta a_{1}{ }^{2} \ll \Delta b_{1}{ }^{2}$.

The two-port scheme owes its success to the law of energy conservation, which dictates that the interference terms between the LO field and the input-signal field contribute with opposite signs to the two outputs of the (lossless) beam splitter and that the noninterference terms contribute with the same sign. The two-port scheme, by using a 50-50 beam splitter and subtracting the two outputs, retains only the interference terms. Its output signal (intensity) is therefore a product of the LO and input-signal mean fields, and its dominant output noise (intensity fluctuations) is due to interference between the input-signal noise $\Delta a_{1}{ }^{2}$ and the LO power and between the LO noise $\Delta b_{1}{ }^{2}$ and the input-signal power. Thus, for large enough LO power, the output noise in the two-port scheme can be made proportional to $\Delta a_{1}^{2}$.

The analysis begins with the beam splitter; assumed linear and lossless with power transmissivity $T$ and re- 


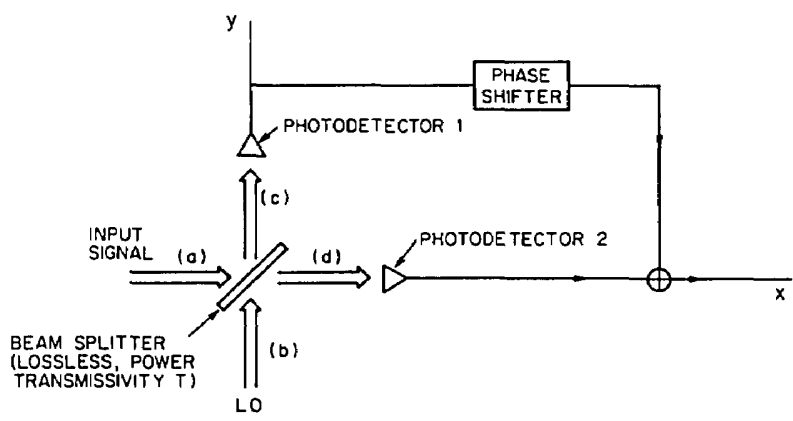

Fig. 1. Yuen-Chan ${ }^{1}$ two-port homodyne detection scheme.

flectivity $1-T$. The law of energy conservation, together with the invariance of a (linear) lossless beam splitter under the combined operations of time reversal and reflection about the $y=-x$ axis, leads to the following matrix transformation between the two in-mode annihilation operators, denoted by $a$ for the signal mode and $b$ for the LO mode, and the two out-mode annihilation operators, denoted by $c$ and $d$ (see Fig. 1):

$$
\begin{aligned}
{\left[\begin{array}{l}
c \\
d
\end{array}\right] } & =U\left[\begin{array}{l}
a \\
b
\end{array}\right], \\
U & =e^{i \Delta}\left[\begin{array}{c}
\sqrt{1-T} e^{i \mu} \\
\sqrt{T}-\sqrt{T}
\end{array} e^{-i \mu}\right]=\left(U^{\dagger}\right)^{-1} .
\end{aligned}
$$

For present purposes, the overall phase $\Delta$ and the relative phase $\mu$-both inherent properties of the beam splitter-need not be known; in fact, the relative phase can effectively be made to take on any desired value by putting phase delays in the path to one or both detectors or by adjusting the relative path lengths.

In order to understand physically the roles played by the various noises as distinct from the mean fields that they accompany, it is useful to separate each annihilation operator into a signal-carrying part and a noisecarrying part. To use the input-signal field as an example, the signal-carrying part is the mean field $\langle a\rangle \equiv$ $A$, a complex number; the noise-carrying part is the annihilation operator minus its mean: $a-\langle a\rangle \equiv \Delta a$. Thus

$$
a=A+\Delta a \equiv A_{1}+i A_{2}+\Delta a_{1}+i \Delta a_{2} .
$$

For simplicity, choose the phase of the LO field so that the quantity $e^{-i \mu}\langle b\rangle$ is equal to the real number $B$; then define the operator

$$
e^{-i \mu} b \equiv B+\Delta b=B+\Delta b_{1}+i \Delta b_{2} .
$$

With this LO phase, homodyning will see only that part of the input signal whose mean is equal to $A_{1}$, so we can consider $A_{2}$ to be zero; thus $A_{1}$ is the signal to be detected, and its inherent noise is characterized by the variance $\Delta a_{1}{ }^{2}$.

Any single-mode state of a radiation field has a noise intensity, a total intensity, and intensity fluctuations (to second order) proportional to these expressions:

$$
\begin{aligned}
\left\langle N_{\bar{a}}\right\rangle \equiv & \left\langle\Delta a^{\dagger} \Delta a\right\rangle=\Delta a_{1}^{2}+\Delta a_{2}^{2}-1 / 2 \\
\left\langle N_{a}\right\rangle \equiv & \left\langle a^{\dagger} a\right\rangle=|A|^{2}+\left\langle N_{\bar{a}}\right) \\
\Delta N_{a}^{2}= & \Delta N_{\bar{a}}^{2}+4\left\langle\left(A_{1} \Delta a_{1}+A_{2} \Delta a_{2}\right)^{2}\right\rangle \\
& +4\left\langle\Delta N_{\bar{a}}\left(A_{1} \Delta a_{1}+A_{2} \Delta a_{2}\right)\right\rangle_{\mathrm{sym}}
\end{aligned}
$$

where the subscript sym means symmetrized. All states of interest have $\left\langle N_{\bar{a}} \Delta a_{1}\right\rangle_{\mathrm{sym}}=\left\langle N_{\bar{a}} \Delta a_{2}\right\rangle_{\mathrm{sym}}=0$. Coherent light with Gaussian excess noise has

$$
\begin{aligned}
\Delta a_{1}{ }^{2}=\Delta a_{2}{ }^{2}=1 / 4 & (2 \bar{n}+1), \quad\left\langle\Delta a_{1} \Delta a_{2}\right\rangle_{\mathrm{sym}}=0, \\
\left\langle N_{\bar{a}}\right\rangle & =\bar{n}, \quad \Delta N_{\bar{a}}^{2}=\bar{n}(\bar{n}+1), \\
\Delta N_{a}{ }^{2} & =|A|^{2}(2 \bar{n}+1)+\bar{n}(\bar{n}+1) \\
& =\left\langle N_{a}\right\rangle+\bar{n}\left(\bar{n}+2|A|^{2}\right) .
\end{aligned}
$$

When $\bar{n}=0$, the above expressions describe a coherent state or the vacuum state $(|A|=0)$. A single-mode squeezed state ${ }^{6-8}$ (with $\varphi=0$ ) has

$$
\begin{aligned}
\Delta{a_{12}}^{2}= & 1 / 4 e^{ \pm 2 r}, \quad\left\langle\Delta a_{1} \Delta a_{2}\right\rangle_{\text {sym }}=0, \\
\left\langle N_{\bar{a}}\right\rangle= & \sinh ^{2} r, \\
\Delta N_{\bar{a}}^{2}= & 1 / 2 \sinh ^{2} 2 r=\left\langle N_{\bar{a}}\right\rangle(1+\cosh 2 r), \\
\Delta N_{a}{ }^{2}= & 1 / 2 \sinh ^{2} 2 r+A_{1}^{2} e^{-2 r}+A_{2}^{2} e^{2 r} \\
= & \left\langle N_{a}\right\rangle+\cosh 2 r \sinh ^{2} r \\
& -2 \sinh r\left(A_{1}^{2} e^{-r}-A_{2}^{2} e^{r}\right) .
\end{aligned}
$$

The beam-splitter outputs are described by the photon-number operators $N_{c} \equiv c^{\dagger} c$ and $N_{d} \equiv d^{\dagger} d$, where

$$
\begin{aligned}
N_{c} & =(1-T) N_{a}+T N_{b}+\sqrt{T(1-T)} P, \\
N_{d} & =T N_{a}+(1-T) N_{b}-\sqrt{T(1-T)} P, \\
P & \equiv 2 \operatorname{Re}\left(e^{-i \mu} b a^{\dagger}\right) \\
& =2\left(A_{1}+\Delta a_{1}\right)\left(B+\Delta b_{1}\right)+2 \Delta a_{2} \Delta b_{2}
\end{aligned}
$$

[Eqs. (2)-(4)]. For the usual situation of a strong LO these expressions reduce to

$$
\begin{aligned}
N_{c} \cong & T B^{2}+2 \sqrt{T(1-T)} A_{1} B \\
& +2 \sqrt{T} B\left(\sqrt{T} \Delta b_{1}+\sqrt{1-T} \Delta a_{1}\right) \\
N_{d} \cong & (1-T) B^{2}-2 \sqrt{T(1-T)} A_{1} B \\
& +2 \sqrt{1-T} B\left(\sqrt{1-T} \Delta b_{1}-\sqrt{T} \Delta a_{1}\right),
\end{aligned}
$$

where here and below $\cong$ means in the strong-LO limit, $(1-T) B^{2} \gg T A_{1}{ }^{2}$. In all the following equations I assume that the power contributed by the fluctuations in the $\mathrm{LO}$ and input-signal fields is negligible compared with the power contributed by the mean fields.

One-port homodyne schemes look only at the output signal $\left\langle N_{d}\right\rangle$ :

$$
\begin{aligned}
\left\langle N_{d}\right\rangle & =\left(\sqrt{1-T} B-\sqrt{T} A_{1}\right)^{2} \\
& \cong(1-T) B^{2}-2 \sqrt{T(1-T)} A_{1} B ;
\end{aligned}
$$

the part of this output that contains input-signal information is

$$
\left\langle N_{d}\right\rangle_{\text {sig }} \cong-2 \sqrt{T(1-T)} A_{1} B .
$$

The output noise in one-port homodyning is given by

$$
\begin{aligned}
\Delta N_{d}{ }^{2} & =4\left(\sqrt{1-T} B-\sqrt{T} A_{1}\right)^{2}\left[(1-T) \Delta b_{1}{ }^{2}\right. \\
& \left.+T \Delta a_{1}^{2}\right]+4\left(\sqrt{1-T} B-\sqrt{T} A_{1}\right)\left[(1-T)^{3 / 2}\right. \\
& \left.\left\langle\Delta N_{\bar{b}} \Delta b_{1}\right\rangle_{\mathrm{sym}}-T^{3 / 2}\left\langle\Delta N_{\bar{a}} \Delta a_{1}\right\rangle_{\mathrm{sym}}\right] \\
& +(1-T)^{2} \Delta N_{\bar{b}}{ }^{2}+T^{2} \Delta N_{\bar{a}}{ }^{2}+4 T(1-T)\langle X\rangle,
\end{aligned}
$$

$$
\begin{aligned}
\langle X\rangle \equiv & \Delta a_{1}^{2} \Delta b_{1}^{2}+\Delta a_{2}^{2} \Delta b_{2}^{2}-1 / 8 \\
& +2\left\langle\Delta a_{1} \Delta a_{2}\right\rangle_{\text {sym }}\left\langle\Delta b_{1} \Delta b_{2}\right\rangle_{\text {sym }} .
\end{aligned}
$$

Neglecting pure noise terms and taking the usual strong-LO limit gives 


$$
\begin{aligned}
\Delta N_{d^{\prime}}{ }^{2}= & 4\left(\sqrt{1-T} B-\sqrt{T} A_{1}\right)^{2}\left[T \Delta a_{1}^{2}\right. \\
& \left.+(1-T) \Delta b_{1}^{2}\right] \\
\cong & \left.4(1-T) B^{2}\left[T \Delta a_{1}{ }^{2}+(1-T) \Delta b_{1}\right] 1 c\right)
\end{aligned}
$$

The SNR for one-port homodyning is defined as the ratio of $\left\langle N_{d}\right\rangle_{\text {sig }}$ to $\left(\Delta N_{d}\right)^{1 / 2}$ :

$$
\begin{aligned}
(\mathrm{SNR})_{1} & \equiv \frac{\left|\left\langle N_{d}\right\rangle\right|_{\text {sig }}}{\left(\Delta N_{d}^{2}\right)^{1 / 2}} \\
& \cong \sqrt{T} \frac{A_{1}}{\left[T \Delta a_{1}^{2}+(1-T) \Delta b_{1}^{2}\right]^{1 / 2}} .
\end{aligned}
$$

Equations (11c) and (12) suggest that the output noise and SNR for one-port homodyning might be made insensitive to $L O$ quadrature-phase noise if $T \cong 1$ with ( 1 $-T) B^{2}$ finite; however, under these conditions other noise sources (those independent of LO power) may become important, so in practice it may not be possible to let $T$ be close to $1 .{ }^{4}$ Regardless of LO and inputsignal power, one-port homodyning is insensitive to $\mathrm{LO}$ quadrature-phase noise only if $(1-T) \Delta b_{1}{ }^{2} \ll$ $T \Delta a_{1}{ }^{2}$.

The two-port scheme suggested by Yuen and Chan ${ }^{4}$ uses a 50-50 beam splitter and looks at the quantity $N_{c}$ $-N_{d}=P$, for which [Eqs. (8c) and (11b)]

$$
\begin{aligned}
\langle P\rangle & =2 B A_{1}, \\
\Delta P^{2} & =4 B^{2} \Delta a_{1}^{2}+4 A_{1}^{2} \Delta b_{1}^{2}+4\langle X\rangle \\
& \cong 4 B^{2} \Delta a_{1}^{2}, \\
(\mathrm{SNR})_{2} & \equiv \frac{\langle P\rangle}{\left(\Delta P^{2}\right)^{1 / 2}} \cong \frac{A_{1}}{\left(\Delta a_{1}^{2}\right)^{1 / 2}} .
\end{aligned}
$$

By adjusting $\theta$ (phase shifter in Fig. 1 ) in the combination $P(\theta) \equiv N_{c} \cos \theta+N_{d} \sin \theta$ of the two photodetector outputs, one can observe $\Delta a_{1}{ }^{2}$ and $\Delta b_{1}{ }^{2}$ individually ( $\theta$ $=\mp \pi / 4$ ) or in any linear combination.

By contrast, direct detection of the same input signal $\left(A_{2} \equiv 0\right)$ would give the following output signal, noise, and SNR [Eqs. (5)]:

$$
\begin{aligned}
\left\langle N_{a}\right\rangle & \cong A_{1}^{2}, \quad \Delta N_{a}^{2} \cong 4 A_{1}^{2} \Delta a_{1}^{2}, \\
(\mathrm{SNR})_{D} & \equiv \frac{\left\langle N_{a}\right\rangle}{\left(\Delta N_{a}^{2}\right)^{1 / 2}} \cong \frac{A_{1}}{2\left(\Delta a_{1}^{2}\right)^{1 / 2}}=1 / 2(\mathrm{SNR})_{2} .
\end{aligned}
$$

For a weak, highly squeezed input signal, in which the power associated with the increased fluctuations of the unsqueezed (conjugate) quadrature rivals the meanfield power, the approximate expressions (14a) must be replaced by the exact expressions [Eqs. (5) and (7)].

The other important source of noise in homodyning is nonideal photodetectors. A photodetector with quantum efficiency $\eta$ can be modeled as a lossless beam splitter with power transmissivity $T \equiv \eta$ followed by an ideal photodetector. The two inputs to the beam splitter are the signal and the vacuum state, described by annihilation operators $d$ and $d_{0}$, respectively. The output of a nonideal photodetector is described by the annihilation operator $d^{\prime}$, where [Eq. (2)]

$$
d^{\prime}=e^{i n}\left(\sqrt{\eta} d-e^{-i \nu} \sqrt{1-\eta} d_{0}\right)
$$

and the phases $\Lambda$ and $\nu$ are inconsequential here. One-port homodyning with a nonideal photodetector gives the following output signal, noise, and SNR:

$\left\langle N_{d^{\prime}}\right\rangle_{\mathrm{sig}}=\eta\left\langle N_{d}\right\rangle_{\mathrm{sig}} \cong-2 \eta \sqrt{T(1-T)} A_{1} B$

$$
\begin{aligned}
\Delta N_{d^{\prime}}{ }^{2}= & \eta^{2} \Delta N_{d^{\prime}}^{2}+\eta(1-\eta)\left\langle N_{a^{\prime}}\right\rangle \\
\cong & (1-T) \eta^{2} B^{2}\left[4 T \Delta a_{1}^{2}\right. \\
& \left.+4(1-T) \Delta b_{1}^{2}+(1-\eta) / \eta\right] \\
(\mathrm{SNR})_{1^{\prime}}= & \frac{2 \sqrt{T} A_{1}}{\left[4 T \Delta a_{1}^{2}+4(1-T) \Delta b_{1}^{2}+(1-\eta) / \eta\right]^{1 / 2}}
\end{aligned}
$$

Two-port homodyning with identical nonideal photodetectors gives this output signal, noise, and SNR:

$$
\begin{aligned}
\left\langle P^{\prime}\right\rangle & =\eta\langle P\rangle=2 \eta B A_{1}, \\
\Delta P^{\prime 2} & =\eta^{2} \Delta P^{2}+\eta(1-\eta)\left[\left\langle N_{c}\right\rangle+\left\langle N_{d}\right\rangle\right] \\
& \cong \eta^{2} B^{2}\left[4 \Delta a_{1}^{2}+(1-\eta) / \eta\right] ; \\
(\mathrm{SNR})_{2^{\prime}} & \cong \frac{2 A_{1}}{\left[4 \Delta a_{1}^{2}+(1-\eta) / \eta\right]^{1 / 2}} \cdot
\end{aligned}
$$

Direct detection of the same input signal would give the following output signal, noise, and SNR:

$$
\begin{aligned}
\left\langle N_{a^{\prime}}\right\rangle & \cong \eta\left\langle N_{a}\right\rangle \cong \eta A_{1}^{2} \\
\Delta N_{a^{\prime}}{ }^{2} & \cong \eta^{2} A_{1}^{2}\left[4 \Delta a_{1}^{2}+(1-\eta) / \eta\right] \\
(\mathrm{SNR})_{D^{\prime}} & \cong \frac{A_{1}}{\left[4 \Delta a_{1}^{2}+(1-\eta) / \eta\right]^{1 / 2}}=1 / 2(\mathrm{SNR})_{2^{\prime}}
\end{aligned}
$$

Equations (16) and (17) show that the absence of any contribution from LO quadrature-phase noise in the output noise of two-port homodyning can be a distinct advantage when one wants to detect squeezing in the input signal. For example, for two-port homodyning with efficient photodetectors $(\eta \cong 0.9)$ the ratio between the output noise produced by a coherent-state input signal ( $\left.4 \Delta \alpha_{1}{ }^{2}=1\right)$ and that produced by an input signal that has been squeezed by a factor of $10\left(4 \Delta a_{1}^{2} \equiv e^{-2 \text { r }}\right.$ $\cong 0.1$ ) is about 5:1. The same ratio for one-port homodyning with an ideal (coherent-state) LO $\left(4 \Delta b_{1}{ }^{2}=\right.$ 1) and a $90 / 10$ beam splitter is about 3.5:1. For the same squeezed input signal, two-port homodyning offers an improvement in output SNR over one-port homodyning by a factor of roughly 1.3 , and it has the additional advantage of not requiring a quiet local oscillator. More-efficient photodetectors and/or a more highly squeezed input signal would make this comparison more dramatic.

This research was supported in part by National Science Foundation Grant AST82-14126.

\section{References}

1. H. P. Yuen and J. H. Shapiro, in Coherence and Quantum Optics IV, L. Mandel and E. Wolf, eds. (Plenum, New York 1978).

2. J. H. Shapiro, H. P. Yuen, and J A. Machado Mata, IEEE Trans. Inf. Theory IT-25, 179 (1979).

3. H. P. Yuen and J. H. Shapiro, IEEE Trans. Inf. Theory IT-26, 78 (1980).

4. H. P. Yuen and V. W. S. Chan, Opt. Lett. 8,177 (1983).

5. G. L. Abbas V. W. S. Chan, and T. K. Yee, Opt. Lett. 8, 419 (1983).

6. H. P. Yuen, Phys. Rev. A 13, 2226 (1976).

7. C. M. Caves, Phys. Rev. D 23, 1693 (1981)

8. B. L. Schumaker and C. M. Caves, in Coherence and Quantum Optics V, L. Mandel and E. Wolf, eds. (Plenum, New York, 1983). 